\title{
Toxin producing Vibrio cholerae O75 outbreak, United States, March to April 2011
}

TJ M Onifade ${ }^{1,2}$, R Hutchinson ${ }^{1,2}$, K Van Zile ${ }^{1,2}$, D Bodager ${ }^{1,2}$, R Baker $^{3}$, C Blackmore (Carina.Blackmore@doh.state.fl.us) ${ }^{1}$

1. Florida Department of Health Bureau of Environmental Health Medicine, United States

2. Food and Waterborne Disease Program, United States

3. Florida Department of Health Bureau of Laboratories, United States

Citation style for this article:

Onifade TM, Hutchinson R, Van Zile K, Bodager D, Baker R, Blackmore C. Toxin producing Vibrio cholerae O75 outbreak, United States, March to April 2011. Euro Surveill. 2011;16(20):pii=19870. Available online: http://www.eurosurveillance.org/ViewArticle.aspx?Articleld=19870

Article published on 19 May 2011

The Florida Department of Health, Florida, United States, is investigating a Vibrio cholerae 075 outbreak. Ten cases with disease onsets from 23 March to 13 April 2011, presented with gastrointestinal symptoms of diarrhoea, nausea, vomiting, cramps, chills, and/or fever, after consuming raw or lightly cooked oysters harvested from Apalachicola Bay, Florida. Symptoms were milder than those during outbreaks of epidemic (serogroup $0_{1}$ and $0_{139}$ ) Vibrio cholerae; no case required rehydration treatment or hospitalisation.

\section{Outbreak report}

On Friday 15 April, 2011 the epidemiology team of the Escambia County Health Department (CHD), Florida, United States (US), notified the Florida Department of Health's Food and Waterborne Disease Program (FWDP) of a case of Vibrio cholerae non-01/non-0139. The man in his early 20 s had fallen ill with cramps, fever, watery diarrhoea, and nausea on 12 April after consuming raw oysters on 6 April in a restaurant. The bacterial isolate was sent to the Florida Department of Health's Bureau of Laboratories (BOL) in Jacksonville for typing and toxin testing. The suspect toxin-producing $V$. cholerae 075 specimen was forwarded to the Centers for Disease Control and Prevention (CDC) and confirmed positive on 19 April.

In the US, the intra- and interstate regulation of oysters is performed by state (Florida Department of Agriculture and Consumer Services, Division of Aquaculture (DOACS)) and federal agencies (US Food and Drug Administration (FDA)), respectively. These agencies require the tracking of oysters through tags that note the harvest date and area. Attempts were made to collect the oyster tags but they were unavailable from the restaurant.

On 18 April, Nassau CHD reported two cases in their late 405 and late 205 respectively, who developed a gastrointestinal illness the day after having purchased live shell stock oysters and consuming them steamed on 10 April. Symptoms included nausea, vomiting, diarrhoea, and chills. One of the patients provided a stool sample that tested positive for toxigenic $V$. cholerae 075 .The other, who had presented with the same symptoms, did not provide samples. This patient was included in our case count as a probable case. The tag for the oysters had been discarded but records at the seafood dealer indicated that they had been harvested from Apalachicola Bay area 1642.

The FWDP was notified of a Louisiana, US $V$. cholerae non-01/non-0139 case on Monday 19 April. The case became ill on 9 April after consuming raw oysters at a restaurant in Okaloosa, Florida, on 7 April. Tags for oysters likely eaten by the case were retrieved from the restaurant on Tuesday 20 April. The oysters had been harvested from the Apalachicola Bay area 1642 on 3 and 6 April. The toxin status for that case is still unconfirmed by CDC so this remains a suspected case.

On Wednesday 20 April, the FWDP investigators contacted the Florida DOACS, the agency with oversight over the Florida oyster industry, notifying them of the $V$. cholerae non-01/non-0139 cluster investigation.

On 21 April, the FWDP coordinator and investigators issued a state-wide alert to the Florida EpiCom system, a state-wide epidemiology electronic alert system, notifying public health officials of the toxigenic $V$. cholerae oyster related case investigation. A similar notification was posted nationally on the CDC EpiX notification system, the federal epidemiology alert system, on 28 April.

Ten cases (eight confirmed, one probable, and one suspect) were identified in this outbreak (Figure 1). Seven were Florida residents, the three other cases were from Indiana, Georgia and Louisiana (Figure 2). The cases ranged from 22 to 74 years of age; six of the ten cases were males. Most cases were in good health, with only one reporting to have pre-existing conditions (kidney problems and a coronary artery stent). Cases reported gastrointestinal symptoms of nausea $(n=7)$, vomiting $(n=4)$, diarrhoea $(n=9)$, chills $(n=8)$, cramps $(n=1)$ and/ 
or fever $(n=1)$ and none required hospitalisation. Dates of exposure ranged from 21 March to 11 April and onset of symptoms occurred between 23 March and 13 April. The average time from exposure to onset of symptoms was 2 days (range: 1 to 6 days). Information gathered during the investigation, which was derived from three sets of oyster tags, implicated one single harvest area in the Apalachicola Bay, Gulf of Mexico, as the source of the contaminated oysters. No additional cases of $V$. cholerae have been reported since 13 April and no oysters harvested later than 6 April have been implicated in any related illnesses.

In response to the outbreak, DOACS conducted an investigation into the implicated oyster harvesting area, Apalachicola Bay area 1642 (Figure 2). The area was closed on 30 April and all dealers and retailers were asked to recall any implicated product still in commerce. The area was reopened to harvesting on 11 May 2011 after DOACS had 15 oyster samples from 10 different sections of the implicated harvest area tested for $V$. cholerae 075 at the FDA laboratory in Dauphin Island, Louisiana. All samples were negative.

\section{Background}

Vibrio spp. bacteria are common in the warm coastal waters of the state of Florida and are also found in local shell fish. Vibrio vulnificus is an important cause of gastrointestinal illness and wound infection in the state, causing 24 cases of illness and six deaths in 2009 [1]. Human illness from native, nontoxigenic non-01 and non-0139 serogroups of $V$. cholerae are reported regularly [2] and all Vibrio diseases are notifiable in Florida. However, despite the favourable ecological conditions, and the fact that Florida receives many travellers from cholera endemic countries, toxigenic $V$. cholerae infections are uncommon. After the 2010 cholera outbreak in Haiti, ten cases of imported $V$. cholerae 01 serotype Ogawa were confirmed in state residents, with no secondary transmission detected (Ann Schmitz, personal communication, 16 April 2011) [3]. The subsequent heightened awareness of cholera has increased the number of human samples submitted to
Florida Department of Health's BOL for $V$. cholerae testing, with more than 40 isolates having been evaluated since November 2010 [4]. At the same time, the turnaround time for test results has also been improved by adding capacity for the detection of cholera toxin. For $V$. cholerae testing, isolates or stools from ill persons, which were previously screened by hospital or private laboratories and suspected $V$. cholerae positive, are referred to the BOL as "suspect $V$. cholerae". Stools are tested by direct thiosulfate citrate bile sucrose agar (TCBS) and enrichment (alkaline peptone water $35^{\circ} \mathrm{C}$ incubation for 8 hours and 18 hours), then subculture to TCBS. Suspect colonies are checked biochemically for the matching $V$. cholerae pattern of reactions and then serology is performed with 01 and 0139 antisera. All $V$. cholerae isolates are also checked for cholera toxin. When isolates do not agglutinate in $0_{1}$ or 0139 antisera but do produce cholera toxin, isolates are referred to CDC and serology for $V$. cholerae 075 is performed. This process takes from 10 to 14 days.

\section{Discussion and conclusions}

Toxigenic $V$. cholerae infections are highly unusual in Florida. This report describes the first $V$. cholerae 075 outbreak detected in the state, between 23 March and 13 April 2011. In Florida, the first isolate of toxigenic $V$. cholerae 075 to be identified was reported in November 2010 , and originated from an immunocompromised Florida resident with a history of oyster consumption. Previously, the serogroup had been associated with sporadic cases of gastrointestinal disease after oyster and other seafood consumption in the south-eastern US [5]. Tobin-D'Angelo et al. [5] describe eight cases with a similar diarrhoeal disease to the ones reported here, who were identified in Georgia, Louisiana, Alabama and South Carolina between 2003 and 2007. These sporadic cases differ from the cases in the outbreak in question here. Four of five cases described in detail had underlying health conditions, whereas only one of the cases in this outbreak had underlying conditions. Two of the eight cases were hospitalised in the analysis of the sporadic cases whereas cases associated with this outbreak experienced milder symptoms

\section{FIGURE 1}

Cases of cholera O75 outbreak from oyster consumption, United States, March-April 2011 (n=10)

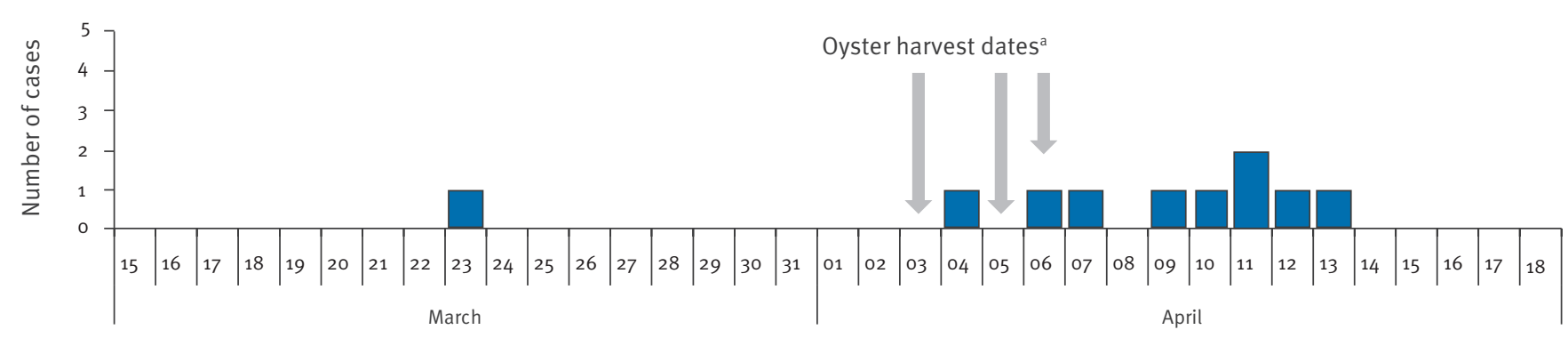

\footnotetext{
${ }^{\mathrm{a}}$ When these dates were available from the oyster tags.
} 
and did not require hospitalisation. Some of these differences may be explained by the increases in testing, which were also the result of recent concerns of imported cholera from Haiti. Persons displaying gastrointestinal symptoms are more likely to have cholera testing done than in the past. Historically, cases with severe illness may have been more likely tested than those with milder symptoms, resulting mainly in severe cases being reported. These factors may have allowed the current detection of this rare serotype of cholera in a relatively healthy population.

Epidemic cholera is an important cause of morbidity and mortality worldwide [6]. Outbreaks generally occur when there are significant deficits in the water sanitation and hygiene infrastructure in the communities, allowing for rapid spread of disease via food and/ or water consumption. Gastrointestinal symptoms are generally mild, however about $20 \%$ of affected individuals develop acute, severe, dehydrating watery diarrhoea which can be fatal if left untreated. The $V$. cholerae 075 appears to cause a milder disease than infection with $V$. cholerae serogroups $\mathrm{O}_{1}$ and $\mathrm{O}_{139}$ and outbreaks in countries with universal access to health care are likely to have limited public health impact. The outbreak reported here is a reminder of the conductive environmental conditions for $V$. cholerae growth in Florida waters and the importance of maintaining sanitary and food safety practices to avoid future outbreaks.

This is the first recorded outbreak related to $V$. cholerae 075 and oyster consumption. The identification of the outbreak could be the result of an increase in

\section{FIGURE 2}

Geographical distribution by state or county of residence of cholera $\mathrm{O} 75$ cases from oyster consumption, and oyster harvesting area, United States, March-April 2011 ( $\mathrm{n}=10)$

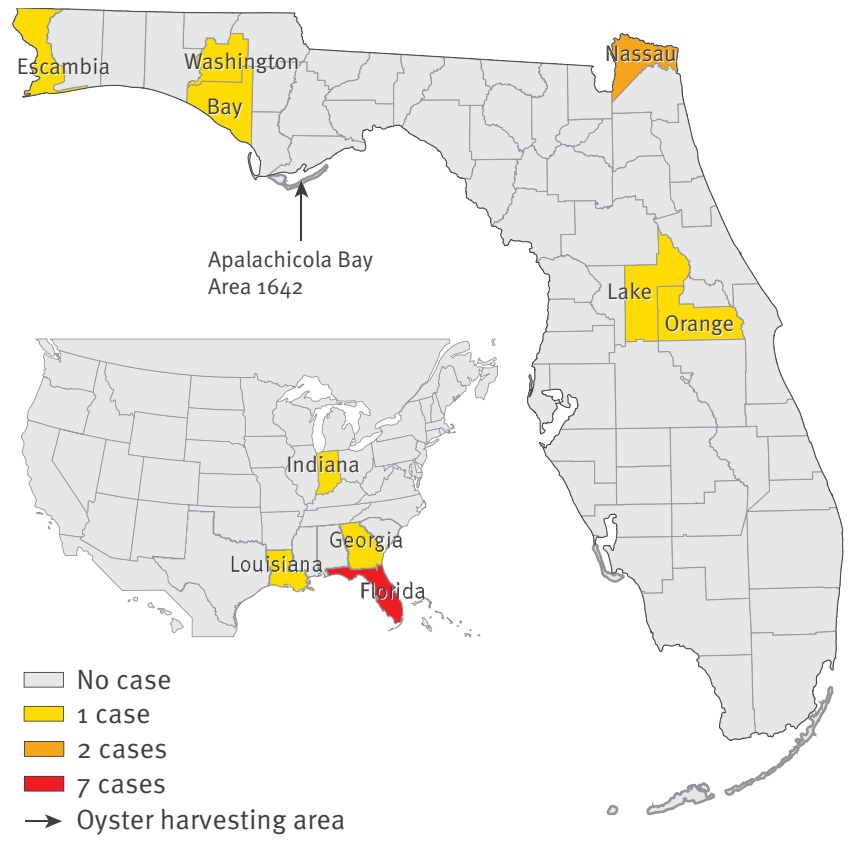

testing for $V$. cholerae in human stool isolates, allowing unprecedented detection of a phenomenon that may have recurrently occurred in the past. Alternatively, the outbreak could be due to some change in the environment, acute or longer term, which has allowed this pathogen to establish or emerge in the oyster population. In the wake of this outbreak, there is concern around the oyster harvesting environment and the regulatory agency, DOACS, is continuing to look into incidents that could have influenced the presence or load of this pathogen in the oyster harvest areas.

Florida is a state of international significance given the large numbers of tourist that visit the state annually and this may raise concern internationally. Here we report preliminary results of the investigation to raise awareness among public health professionals worldwide about this rare oyster related $V$. cholerae outbreak. We will be monitoring for future cases of $V$. cholerae 075 , and will continue our investigation into other factors associated with this outbreak.

\section{Acknowledgments}

The authors of this outbreak report would like to thank the Florida Department of Health's Bureaus of Laboratories and Epidemiology, the epidemiology and environmental health staff of Nassau, Lake, Bay, Escambia, Orange, and Washington County Health Departments, the Florida Department of Agriculture and Consumer Services Division of Aquaculture and the Waterborne Disease Prevention Branch at Centers for Disease Control and Prevention.

\section{References}

1. State of Florida Department of Health. Florida Morbidity Statistics Report 2009. Tallahassee (FL): 2010 Dec. Available from: http://www.doh.state.fl.us/disease_ctrl/epi/Morbidity Report/2009/AMR_\%202009.pdf

2. Weis KE, Hammond RM, Hutchinson R, Blackmore CG. Vibrio illness in Florida 1998-2007. Epidemiol Infect. 2011:139(4):591-8.

3. Jenkins PH, Montejano HJ, Abbasi MJ, Crowley MS, O’Brien MG; Conte V, et al. Update on Cholera - Haiti, Dominican Republic, and Florida, 2010, MMWR Morb Mortal Wkly Rep. 2010;59(50):1637-41.

4. Florida Department of Health. Guidance for Haiti-related Toxigenic Vibrio cholerae Type $0_{1}$, Case Reporting for County Health Departments. [Accessed 15 May 2011]. Available from: http://www.doh.state.fl.us/disease_ctrl/epi/acute/ haiti_cholera_impact_surv_guidance_chds_v1.1.pdf

5. Tobin-D'Angelo M, Smith AR, Bulens SN, Thomas S, Hodel $\mathrm{M}$, Izumiya $\mathrm{H}$ et al. Severe diarrhea caused by cholera toxin-producing vibrio cholerae serogroup 075 infections acquired in the southeastern United States. Clin Infect Dis. 2008;47(8):1035-40.

6. World Health Organization (WHO). Cholera. Fact sheet No 107. June 2010. [Accessed 16 May 2011]. Available from: http:// www.who.int/mediacentre/factsheets/fs107/en/ 